Pacific Journal of Mathematics

TRANSLATION INVARIANT MEASURE OVER SEPARABLE
HILERT SPACE AND OTHER TRANSLATION SPACES 


\title{
TRANSLATION INVARIANT MEASURE OVER SEPARABLE HILBERT SPACE AND OTHER TRANSLATION SPACES
}

\author{
F. H. BROWNELL
}

1. Introduction. We consider the problem of defining a nontrivial, translation-invariant Borel measure over real separable Hilbert space. As noted by Loewner [4], this is not possible; but instead of relinquishing as he does the real number system for a non-Archimedean ordered field for the values of a "measure," we shall consider several topological subspaces of Hilbert space arising frequently in analysis. These are locally compact; and using either the Kolmogoroff stochastic processes construction [2], or else following the Haar measure construction [1] or [5], we can get a nontrivial, essentially translationinvariant Borel measure. However, since the special subspaces considered are not groups under translation, and do not even contain a group germ, the usual Haar measure construction must be modified in a special fashion, and the precise translation invariance obtained is somewhat restrictive. Actually we carry through this modified Haar measure construction for the more general situation of a locally compact translation space, which is defined as an appropriate subspace of an Abelian topological group. The results are collected in a summary at the end.

2. Formulation of the problem. Let

$$
\ell_{2}=\left\{x=\left\{x_{n}\right\} \mid \sum_{n=1}^{\infty}\left(x_{n}\right)^{2}<+\infty, x_{n} \text { real }\right\},
$$

the square summable real sequences and thus the real separable Hilbert space prototype. Since $\ell_{2}$ is a subset of $R_{\infty}$, the countably infinite Cartesian product of the real line $(-\infty, \infty)$, we have available on $\ell_{2}$ as well as the $\ell_{2}$ norm metric topology also the product topology defined relatively from $R_{\infty}$. Under these two topologies we shall consider the $l_{2}$-subsets

$$
X=\left\{x \in \ell_{2}|| x_{n} \mid \leq h(n) \text { for all } n\right\},
$$

Received August 4, 1951.

Pacific J. Math. 3 (1953), 531-553 


$$
Y=\left\{\left.x \in \ell_{2}\left|\sum_{j=n}^{\infty}\right| x_{j}\right|^{2} \leq f(n) \text { for all } n\right\}
$$

where $f(n)$ and $h(n)$ are specified functions defined over the integers $n \geq 1$ with values real or $+\infty$ having $h(n)>0$ and $f(n) \geq f(n+1)>0$.

Let $Z=X$ or $Y$; we want to define the Borel class of subsets of $Z$. The open intervals of $Z$ are defined relatively from the elementary open intervals of $R_{\infty}$, and so we can define $B_{1}$ as the $\sigma$-algebra of subsets of $Z$ generated by the open intervals, $B_{2}$ as that generated by the product-topology open sets, $B_{3}$ by the metric spheres, and $B_{4}$ by the metricly open sets. Actually $B_{1}=B_{2}=B_{3}=B_{4}$, and will be denoted by $B$ and called the class of Borel subsets of $Z$. To see this we note first by using the rationals that $R_{\infty}$ and hence $Z$ has a countable basis of open intervals, so $B_{1}=B_{2}$. Similarly $B_{3}=B_{4}$, since $\ell_{2}$ and hence $Z$ is a separable metric space and thus has a countable basis of spheres. Since any product-topology open set is clearly open metricly, $B_{2} \subseteq B_{4}$. Now it is easy to see that any closed sphere

$$
S=\{x \in Z \mid\|x-y\| \leq \rho\}
$$

is actually closed in the product topology. Since any open sphere is a countable union of closed ones, $B_{3} \subseteq B_{2}$. Thus $B_{3}=B_{4}$ makes $B_{1}=B_{2}=B_{3}=B_{4}$, as desired.

Define

$$
[A+u]=\left\{x \in R_{\infty} \mid(x-u) \in A\right\}
$$

for $u \in R_{\infty}$ and for any subset $A$ of $R_{\infty}$. We note that $u \in Z$ and $A \subseteq Z$ do not always make $[A+u] \subseteq Z$ if $Z \neq \ell_{2}$. However, if $A \in B$ and $u \bar{\epsilon} R_{\infty}$ then $[A+u] \cap Z \in$ B. For

$$
\mathcal{F}=\{A \mid[A+u] \cap Z \in B\}
$$

is easily seen to be a $\sigma$-algebra containing the intervals of $Z$, so $B=B_{1} \subseteq \stackrel{7}{\mathcal{C}}$, which gives the result.

Our problem is to find a Borel measure $\phi$, that is, a nonnegative extended real set function defined and countably additive over $B$, which is nontrivial (Condition I) and translation-invariant (Condition II or $\mathrm{II}^{\prime}$ ) according to a specified topology.

CONDITION I. $\phi(Z)>0$ and $\phi(V)<+\infty$ for some nonempty $V$ open in the specified topology; 
Condition II. $\phi([A+u])=\phi(A)$ if $A \in B, u \in \ell_{2}$, and $[A+u] \subseteq Z$;

Condition II'. a) $\phi([A+u])=\phi(A)$ if $A \in B, u \in \ell_{2}, A \subseteq V$, where $V$ and $[V+u]$ are both open subsets of $Z$.

b) $\phi([A+u] \cap Z) \leq \phi(A)$ if $u \in \ell_{2}$ and both $A$ and $[A+u] \cap Z$ are open subsets of $Z$.

Condition II clearly implies II' $^{\prime}$, and hence is a stronger requirement.

3. Negative results. We shall start with a few preliminary lemmas. First define

$$
S(Z, x, \rho)=\{y \in Z \mid\|x-y\|<\rho\},
$$

the $\rho$-radius open $Z$-sphere about $x$.

LEMMA 1. For any real $r>0$ there exists no nonnegative, finitely additive set function $\phi$ over the Borel subsets of

$$
Z=Y=\overline{S\left(\ell_{2}, 0, r\right)}
$$

satisfying II', (or thus II also), under the metric topology such that

$$
0<\phi\left(S\left(\ell_{2}, 0, \rho\right)\right)<+\infty \text { for } 0<\rho \leq r \text {. }
$$

Proof. Let

$$
p^{x}=\left\{_{p} x_{j}\right\} \in S\left(\ell_{2}, 0, r\right)
$$

by defining $p^{x_{j}}=0$ if $j \neq p$ and $p_{p}=r / 2$ for integer $p \geq 1$. Let

$$
V_{p}=S\left(\ell_{2}, p x, \frac{1}{4} r\right)
$$

so that $V_{p} \subseteq S\left(\ell_{2}, 0, r\right)$; and $V_{p} \cap V_{q}=\phi$ for $p \neq q$ follows from

$$
\left\|y-y^{\prime}\right\| \geq\left\|p_{p} x-q^{x}\right\|-2\left(\frac{1}{4} r\right)=\frac{\sqrt{2}-1}{2} r>0
$$

for $y \in V_{p}$ and $y^{\prime} \in V_{q}$. But $\mathrm{II}^{\prime}$ under the metric topology makes

$$
\phi\left(V_{p}\right)=\phi\left(S\left(\ell_{2}, 0, \frac{1}{4} r\right)\right)=b
$$


with $0<b<+\infty$. Thus

$$
S\left(\ell_{2}, 0, r\right) \supset \underset{p=1}{U} V_{p},
$$

and finite additivity of $\phi$ yields the contradiction

$$
0<N b=\sum_{p=1}^{N} \phi\left(V_{p}\right) \leq \phi\left(S\left(l_{2}, 0, r\right)\right)<+\infty
$$

for arbitrary integer $N$. Thus such $\phi$ cannot exist.

LemMa 2. If

$$
0<\inf _{n \geq 1} h(n) \text { for } Z=X
$$

or if

$$
0<\inf _{n \geq 1} f(n) \text { for } Z=Y \text {, }
$$

then for any $x \in Z$ and $\rho>0$ there exists some $z \in Z$ and $\rho^{\prime}>0$ such that $S\left(\ell_{2}, z, \rho^{\prime}\right) \subseteq S(Z, x, \rho)$.

Proof. For the given $x \in Z$ choose some $N \geq 1$ so that

$$
\sum_{j=N+1}^{\infty}\left(x_{j}\right)^{2} \leq\left(\frac{1}{3} \rho\right)^{2},
$$

possible since $x \in \ell_{2}$. Define

$$
y^{\prime}=\left(y_{1}, \cdots, y_{N}\right)=P(y) \in E_{N}
$$

as the projection of $\ell_{2}$ onto Euclidean $N$ space $E_{N}$. Clearly $P(Z)$ is a convex set with a nonvoid interior in $E_{N}$ including the origin; so we can find an interior point $z^{\prime}$ on the line-segment from $x^{\prime}=P(x)$ to the origin so that

$$
\sum_{n=1}^{N}\left(z_{n}-x_{n}\right)^{2}<\left(\frac{1}{3} \rho\right)^{2}
$$

Define $z \in \ell_{2}$ so that $z^{\prime}=P(z)$ by taking $z_{n}=0$ for $n \geq N+1$. Thus 


$$
\|x-z\|=\left[\sum_{n=1}^{N}\left(z_{n}-x_{n}\right)^{2}+\sum_{n=N+1}^{\infty} x_{n}^{2}\right]^{1 / 2}<\frac{\sqrt{2}}{3} \rho .
$$

Let

$$
b_{0}=\inf _{n \geq 1} h(n)>0 \text { for } Z=X,
$$

or

$$
j_{0}=\left[\inf _{n \geq 1} f(n)\right]^{1 / 2}>0 \text { for } Y \text {. }
$$

Now if $Z=X$, by choosing $\rho^{\prime \prime}>0$ so that $\rho^{\prime \prime}<b_{0}$ and

$$
S\left(E_{N}, z^{\prime}, \rho^{\prime \prime}\right) \subseteq P(Z),
$$

as we may since $z^{\prime} \in$ int $P(Z)$, we get

$$
S\left(\ell_{2}, z, \rho^{\prime \prime}\right) \subseteq Z
$$

If $Z=Y$, then $z^{\prime} \in$ int $P(Z)$ makes

$$
\sum_{j=n}^{N}\left(z_{j}\right)^{2}<f(n)
$$

for $1 \leq n \leq N$, so here we choose $0<\rho^{\prime \prime}<b_{0}$ and

$$
\rho^{\prime \prime}<\min _{1 \leq n \leq N}\left([f(n)]^{1 / 2}-\left[\sum_{j=n}^{N}\left(z_{j}\right)^{2}\right]^{1 / 2}\right) .
$$

Thus

$$
\left[\sum_{j=n}^{\infty}\left(y_{j}\right)^{2}\right]^{1 / 2} \leq\|y-z\|+\left[\sum_{j=n}^{N}\left(z_{j}\right)^{2}\right]^{1 / 2}<f(n) \text { for } 1 \leq n \leq N,
$$

and

$$
\left[\sum_{j=n}^{\infty}\left(y_{j}\right)^{2}\right]^{1 / 2} \leq\|y-z\|<b_{0} \leq f(n) \text { for } n \geq N+1 \text {, }
$$


makes $S\left(\ell_{2}, z, \rho^{\prime \prime}\right) \subseteq Y=Z$.

Thus

$$
\rho^{\prime}=\min \left(\rho^{\prime \prime}, \frac{3-\sqrt{2}}{3} \rho\right)>0
$$

yields

$$
S\left(\ell_{2}, z, \rho^{\prime}\right) \subseteq Z \cap S\left(\ell_{2}, x, \rho\right)=S(Z, x, \rho)
$$

as desired, since

$$
\|y-z\|<\frac{3-\sqrt{2}}{3} \rho
$$

makes

$$
\|x-y\| \leq\|y-z\|+\|x-z\|<\rho
$$

because $\|x-z\|<(\sqrt{2} / 3) \rho$.

THEOREM 3. If

$$
0<\liminf _{n \rightarrow \infty} h(n) \text { with } Z=X,
$$

or if

$$
0<\liminf _{n \rightarrow \infty} f(n) \text { with } Z=Y,
$$

then there exists no Borel measure $\phi$ on such $Z$ which is nontrivial (I) and translation-invariant ( $\left.\mathrm{II}^{\prime}\right)$ under the norm-metric topology.

Proof: Set

$$
b_{0}=\inf _{n \geq 1} h(n) \text { if } Z=X,
$$

or

$$
b_{0}=\left[\inf _{n \geq 1} f(n)\right]^{1 / 2} \text { if } Z=Y
$$

thus clearly $b_{0}>0$ is required by hypothesis. Obviously 


$$
S(Z, 0, \rho)=S\left(\ell_{2}, 0, \rho\right)
$$

for $0<\rho \leq b_{0}$, so the metricly open set

$$
S(Z, x, \rho)=\left[S\left(\ell_{2}, 0, \rho\right)+x\right] \cap Z=[S(Z, 0, \rho)+x] \cap Z
$$

for such $\rho$. Hence if $\phi$ exists, then $\phi(S(Z, x, \rho)) \leq \phi(S(Z, 0, \rho))$ by Condition II'b) for $x \in \ell_{2}, 0<\rho \leq b_{0}$.

Now set

$$
b_{1}=\inf \{\text { all } \rho>0 \text { such that } \phi(S(Z, 0, \rho))>0\} \text {, }
$$

so $\phi(S(Z, 0, \rho))>0$ for $\rho>b_{1}$, and $=0$ for $0<\rho<b_{1}$ if $b_{1}>0$. Actually $b_{1}=0$. For if not set $\delta=\left(\min b_{0}, b_{1} / 2\right)$; then $Z$, being separable, is a countable union of spheres of radius $\rho \leq \delta$. But such spheres have

$$
\phi(S(Z, x, \rho)) \leq \phi(S(Z, 0, \rho))=0,
$$

implying $\phi(Z)=0$ by countable additivity, which contradicts Condition I. Thus $b_{1}=0$ and $\phi(S(Z, 0, \rho))>0$ for all $\rho>0$.

We want to show that $\phi(S(Z, 0, r))<+\infty$ for some $r>0$. By Condition I under the metric topology and Lemma 2 it is clear that there exists some $r>0$ and $z \in Z$ such that

$$
S\left(\ell_{2}, z, r\right) \subseteq Z \text { and } \phi\left(S\left(\ell_{2}, z, r\right)\right)<+\infty .
$$

Since $S\left(\ell_{2}, z, r\right) \subseteq Z$, it is easily seen for either $X=Z$ or $Y=Z$ that we must have $r \leq b_{0}$, and hence

$$
Z \supseteq S\left(\ell_{2}, 0, r\right)=S(Z, 0, r) .
$$

Thus $[S(Z, 0, r)+z]=S\left(\ell_{2}, z, r\right)$, an open subset of $Z$, so Condition II'a) makes

$$
\phi(S(Z, 0, r))=\phi\left(S\left(\ell_{2}, z, r\right)\right)<+\infty \text {. }
$$

Thus

$$
0<\phi(S(Z, 0, \rho))<+\infty
$$

with $S(Z, 0, \rho)=S\left(\ell_{2}, 0, \rho\right)$ for $0<\rho \leq r$ for some $r, 0<r<b_{0}$, which is impossible by Lemma 1 . Thus the stated $\phi$ cannot exist. 
We also easily get the following considerably weaker result for the product topology.

THEOREM 4. If $\{n \mid h(n)=+\infty\}$ is an infinite set, then there exists no Borel measure $\phi$ on $X$ which is nontrivial (I) and translation-invariant (II') under the product topology.

Proof. Let $V$ be any nonempty open interval of $X$. It is clear that by translating along each of the finite set of coordinates given in the definition of the interval $V$, we can find a finite or countable set of ${ }_{p} x \in \ell_{2}$ such that

$$
\left[V+p^{x}\right] \subseteq X \text { and } X=\bigcup_{p=1}\left[V+p^{x}\right]
$$

Also Condition $\left.\mathrm{II}^{\prime} \mathrm{a}\right)$ makes $\phi\left(V+p^{x}\right)=\phi(V)$ if $\phi$ exists. Thus $\phi(X)>0$ for nontriviality yields by countable additivity $\phi(V)>0$ for any open intervall $V \neq \phi$.

Now Condition I under the product topology implies that some open interval $V_{0} \neq \phi$ has $\phi\left(V_{0}\right)<+\infty$, so $0<\phi\left(V_{0}\right)<+\infty$. Since $V_{0}$ is defined in terms of only a finite number of coordinates, and $\{n \mid h(n)=+\infty\}$ is infinite, there must exist some $p$ so that $x \in V_{0}$ imposes no restriction on the $p$ th coordinate of $x$. Let

$$
W_{0}=\left\{y \in V_{0}|| y_{p} \mid<1\right\} \text {. }
$$

a nonvoid open $X$ interval, so $\phi\left(W_{0}\right)>0$. Let ${ }_{0} z_{j}=0$ if $j \neq p,{ }_{0} z_{p}=1$, so clearly $\left\{\left[W_{0}+m_{0} z\right]\right\}$ form a disjoint union of sets $\subseteq V_{0}$ for different integer $m$, with

$$
\phi\left(\left[W_{0}+m_{0} z\right]\right)=\phi\left(W_{0}\right)
$$

by Condition II'a). Thus

$$
+\infty=\sum_{m=1}^{\infty} \phi\left(\mathbb{W}_{0}\right)=\phi\left(\bigcup_{m=1}^{\infty}\left[W_{0}+m_{0} z\right]\right) \leq \phi\left(V_{0}\right)<+\infty,
$$

which is a contradiction. Thus $\phi$ cannot exist.

We remark that $\ell_{2}=X$ by taking $h(n) \equiv+\infty$, so Theorems 3 and 4 show that there exists no Borel measure $\phi$ on $l_{2}$ which is nontrivial and translationinvariant under either the norm metric or product topologies. 
4. Positive results via Kolmogoroff. We want to give conditions under which an invariant measure does exist on $X$ or $Y$, getting a converse of Theorem 3. For $X$ we shall use the construction of Kolmogoroff $[2, \mathrm{p} .27]$ of a probability measure $P$ over real product spaces, in our case $R_{\infty}$. Here we need a family $Q$ of real set functions, each member $Q_{n_{1}}, \cdots, n_{k}$ being nonnegative and countably additive over the intervals of $E_{k}$, with coordinates indexed $n_{1}, \cdots, n_{k}$, and having $Q_{n_{1}}, \cdots, n_{k}\left(E_{k}\right)=1$. The family $Q$ is assumed to satisfy Kolmogoroff's two consistency conditions:

$$
\begin{aligned}
& Q_{n_{1}}, \cdots, n_{k}\left(-\infty,+\infty ; a_{2}, b_{2} ; \cdots ; a_{k}, b_{k}\right)=Q_{n_{2}}, \cdots, n_{k}\left(a_{2}, b_{2} ; \cdots ; a_{k}, b_{k}\right), \\
& Q_{n_{1}}, \cdots, n_{k}\left(a_{1}, b_{1} ; \cdots ; a_{k}, b_{k}\right)=Q_{n_{1}^{\prime}, \cdots, n_{k}^{\prime}}\left(a_{1}^{\prime}, b_{1}^{\prime} ; \cdots ; a_{k}^{\prime}, b_{k}^{\prime}\right),
\end{aligned}
$$

where $n_{i}^{\prime}=n_{j}, a_{i}^{\prime}=a_{j}, b_{i}^{\prime}=b_{j}$ for $n_{1}^{\prime}, \cdots, n_{k}^{\prime}$ a reordering of $n_{1}, \cdots, n_{k}$. The resulting $P$ has $P(I)=Q(\tilde{I})$ if the interval $I$ is the cylinder set by $n_{1}, \cdots, n_{k}$ of the interval $\tilde{I}$ of $E_{k}, P$ being the Borel-Hopf extension $[1, \mathrm{p} .54]$ of $Q$ from the intervals to the Borel sets.

THEOREM 5. If

$$
\sum_{n=N+1}^{\infty}[h(n)]^{2}<+\infty
$$

for some finite $N$, then for $X$ the product and metric topologies coincide, $X$ being locally compact; there exists a Borel measure $\phi$ which is nontrivial (I) and translation-invariant (II) on $X$; and such a measure is unique up to constant factors.

Proof. The stated condition on $h(n)$ makes the equivalence of the topologies over $X$ obvious, as well as local compactness. Let $X^{\prime}, \ell_{2}^{\prime}$, and $R_{\infty}^{\prime}$ be defined like $X, \ell_{2}$, and $R_{\infty}$, except only with coordinates of $n \geq N+1$, so clearly

$$
X=A_{N} \times X^{\prime}
$$

where $A_{N}$ is an interval of $E_{N}$. Construct the Borel measure $P^{*}$ on $R_{\infty}^{\prime}$ by the Kolmogoroff construction from

$$
Q_{n_{1}, \cdots, n_{k}}\left(a_{1}, b_{1} ; \cdots ; a_{k}, b_{k}\right)=\prod_{j=1}^{k} \frac{1}{2 h\left(n_{j}\right)} E\left(n_{j}, a_{j}, b_{j}\right),
$$


where $E(n, a, b)$ is the length, possibly zero, of the interval of intersection of $[-h(n), h(n)]$ and $[a, b]$. This $Q$-function family has $Q_{n_{1}}, \cdots, n_{k}\left(E_{k}\right)=1$, has $Q$ countably additive since it is a multiple of $k$ dimensional Lebesque measure, and satisfies Kolmogoroff's consistency conditions as needed.

Let

$$
V_{p}=\left\{x \in R_{\infty}^{\prime}|| x_{P} \mid>h(p)\right\}
$$

open in $R_{\infty}^{\prime}$; clearly

$$
P^{*}\left(V_{P}\right)=Q\left(\tilde{V}_{P}\right)=\frac{1}{2 h(p)}[E(p,-\infty,-h(p))+E(p, h(p),+\infty)]=0 .
$$

Now

$$
X^{\prime}=\left\{x \in \ell_{2}^{\prime}|| x_{n} \mid \leq h(n) \text { for } n \geq N+1\right\}
$$

and the given condition on $h(n)$ makes it possible to replace $\ell_{2}^{\prime}$ by $R_{\infty}^{\prime}$ in this formula, so that

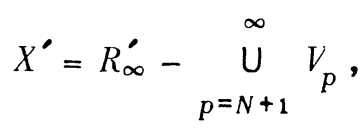

which is in the Borel family $B^{*}$ of $R_{\infty}^{\prime}$. Thus $P^{*}\left(X^{\prime}\right)=P^{*}\left(R_{\infty}^{\prime}\right)=1$ follows from $P^{*}\left(V_{P}\right)=0$, and $X^{\prime}$ is thick in $R_{\infty}^{\prime}$ (see $\left.[1, \mathrm{p} .74]\right)$. Hence $P\left(A \cap X^{\prime}\right)=$ $P^{*}(A)$ defines $P$ uniquely over sets $A \cap X^{\prime}, A \in B^{*}$, which form the Borel family $B$ of $X^{\prime}$, so $P$ is a Borel probability measure on $X^{\prime}$ with $P\left(I \cap X^{\prime}\right)=$ $Q(\tilde{I})$.

Of $\mu_{N}$ is $N$-dimensional Lebesque measure, $\phi=\mu_{N} \times P$ is a Borel measure on $A_{N} \times X^{\prime}=X$. Also

$$
\phi(X)=\mu_{N}\left(A_{N}\right)>0,
$$

and we obtain

$$
\phi\left(B \times X^{\prime}\right)=\mu_{N}(B)<+\infty
$$

for open bounded $E_{N}$ intervals $B \subseteq A_{N}$ by using $P\left(X^{\prime}\right)=1$, and thus $\phi$ is nontrivial $(I)$ on $X$. 
We want to show $\phi$ to be translation-invariant (II) on $X$. If $W$ is any $X$ interval, then $W=X \cap I$ with $I$ an $R_{\infty}$-interval, and if $u \in \ell_{2}$, set

$$
\begin{gathered}
B_{p}=\left\{x \in R_{\infty}|| x_{p} \mid \leq h(p)\right\}, \\
C_{n}=\operatorname{In}\left(\bigcap_{p=1}^{n}\left[B_{p}-u\right]\right) \cap X,
\end{gathered}
$$

and

$$
D_{n}=[I+u] \cap X \cap\left(\bigcap_{p=1}^{n}\left[B_{p}+u\right]\right) \text {, }
$$

so that

$$
\phi(\mathbb{W} \cap[X-u])=\phi(I \cap[X-u] n X)=\lim _{n \rightarrow \infty} \phi\left(C_{n}\right)
$$

and

$$
\phi([W+u] \cap X)=\phi([I+u] \cap X \cap[X+u])=\lim _{n \rightarrow \infty} \phi\left(D_{n}\right) .
$$

Now the first $n$ coordinate edges of $D_{n}$ are those of $C_{n}$ translated by the corresponding $u$ coordinates. Thus taking $n>$ the greatest of the finite number of coordinate indices involved in $l$, from $\phi=\mu_{N} \times P$ and $P\left(X^{\bullet} \cap J\right)=Q(\tilde{J})$ we get $\phi\left(C_{n}\right)=\phi\left(D_{n}\right)$, both being the product of a normalization factor and the first $n$ coordinate edge lengths. Thus we have

$$
\phi(\mathbb{W} \cap[X-u])=\lim _{n \rightarrow \infty} \phi\left(C_{n}\right)=\lim _{n \rightarrow \infty} \phi\left(D_{n}\right)=\phi([W+u] \cap X),
$$

as desired.

Now let $[A+u] \subseteq X$ be given for some Borel subset $A$ of $X$. If $\left\{W_{i}\right\}$ is a countable disjoint $X$-interval family covering $A$, then also

$$
A \subseteq \underset{i}{\bigcup}\left(W_{i} \cap[X-u]\right) \subseteq \underset{i}{\bigcup} W_{i}
$$

Since

$$
\phi(A)=\inf _{A \subseteq \cup_{i}^{u} \mathbb{W}_{i}}\left[\Sigma_{i} \phi\left(\mathbb{W}_{i}\right)\right]
$$


as the unique Borel-Hopf extension $[1, \mathrm{p} .54]$ of $\phi$ from the intervals to the Borel sets, we have

$$
\begin{aligned}
\phi(A) & =\inf _{A \subseteq \underset{i}{u} \mathbb{W}_{i}}\left(\Sigma_{i} \phi\left(\mathbb{W}_{i} \cap[X-u]\right)\right) \\
& =\inf _{A \subseteq \bigcup_{i} \mathbb{W}_{i}}\left(\Sigma_{i} \phi\left(\left[W_{i}+u\right] \cap X\right)\right) \geq \phi([A+u])
\end{aligned}
$$

from

$$
\phi\left(W_{i} \cap[X-u]\right)=\phi\left(\left[W_{i}+u\right] \cap X\right) .
$$

Thus $\phi(A) \geq \phi([A+u])$, and symmetrically $\phi([A+u]) \geq \phi(A)$, so that $\phi(A)=\phi([A+u])$ for Condition II of translation-invariance.

Finally for the uniqueness of $\phi$ it is easy to see by division of intervals into large numbers of equal subintervals that any nontrivial, translation-invariant $\psi$ will have $\psi(I), I$ being an interval of $X$, proportional to the length of each of the edges of $I$. By our definition of $\mu_{N}$ and $Q$, this makes $\psi(I)=K \phi(I)$, with $0<K<+\infty$ and $K$ independent of $I$. The extension to all Borel sets thus gives $\psi(A)=K \phi(A), A \in B$, as desired.

5. Haar measure and translation spaces. For the space $Y$ our positive result is a complete converse of Theorem 3. We shall get the result by considering a considerably more general situation. Let the Hausdorff space $R$ be an Abelian topological group, and as before define

$$
[A+u]=\{x \in R \mid(x-u) \in A\}
$$

under $R$-group addition for $A \subseteq R$ and $u \in R$. Consider a fixed closed subset $Z$ of $R$, which becomes a Hausdorff space under the relative topology from $R$, but not in general a group under $R$-group addition. Such a space containing the zero of $R$ is said to be a translation space if it satisfies the following condition:

i) If $V$ is any open subset of $Z$ containing zero, then $Z$ is covered by the open interiors in $Z$ of the sets of the collection $\{Z n[V+u] \mid u \in R\}$.

Lemma 6. $X$ is a translation space for $R=\ell_{2}$ under the metric topology.

Proof. Let $V$ be the given neighborhood of zero, so that we have some small $\rho>0$ with $S(Z, 0, \rho) \subseteq V$. Then for any given $z \in Z=X$ we will find 
$u \in Z$ and $\rho^{\prime}>0$ so that

$$
S\left(Z, z, \rho^{\prime}\right) \subseteq Z \cap[S(Z, 0, \rho)+u] \subseteq Z \cap[V+u],
$$

which makes $z \in$ int $(Z \cap[V+u])$ for Condition i). First since the given $z \in \ell_{2}$, we can find finite $N$ so that

$$
\left(\sum_{n=N+1}^{\infty} z_{n}^{2}\right)^{1 / 2}<\frac{1}{2} \rho,
$$

and then define $u \in Z=X$ by $u_{n}=z_{n}$ for $1 \leq n \leq N$ and $u_{n}=0$ for $n>N$. Then set

$$
\rho^{\prime}=\min \left(\frac{1}{2} \rho, h(n) \text { for } n=1,2, \cdots, N\right)>0 \text {, }
$$

so any $x \in S\left(Z, z, \rho^{\prime}\right)$ has

$$
\|x-u\| \leq\|x-z\|+\|z-u\|<\rho^{\prime}+\frac{1}{2} \rho \leq \rho .
$$

Any such $x$ also has

$$
\left|x_{n}-u_{n}\right|=\left|x_{n}-z_{n}\right|<\rho^{\prime} \leq \dot{h}(n)
$$

for $1 \leq n \leq N$, and

$$
\left|x_{n}-u_{n}\right|=\left|x_{n}\right| \leq h(n)
$$

for $n>N$, so that $x \in[S(Z, 0, \rho)+u]$. Thus

$$
S\left(Z, z, \rho^{\prime}\right) \subseteq Z \cap[S(Z, 0, \rho)+u]
$$

as desired.

Lemma 7. $Y$ is a translation space for $R=\ell_{2}$ under the metric topology.

Proof. If $V$ is the given neighborhood of zero in $Z=Y$, we can find $\rho>0$ with $\rho^{2}<f(1)$ and $S(Z, 0, \rho) \subseteq V$. Now either $\rho^{2} \leq f(n)$ for all $n$, or else by the definition of $Y$ there is a unique finite $N$ with

$$
f(N) \geq \rho^{2}>f(N+1) .
$$


In the first case for the given $z \in Z$ we take $u=z$, and since now $S(Y, 0, \rho)=$ $S\left(\ell_{2}, 0, \rho\right)$ by $\rho^{2} \leq f(n)$, we have

$$
S(Z, z, \rho)=Z \cap\left[S\left(\ell_{2}, 0, \rho\right)+u\right] \subseteq Z \cap[V+u]
$$

for $z \in$ int $(Z \cap[V+u])$ as desired for Condition i ).

In the second case for the given $z \in Z=Y$ we define $u \in Z$ by $u_{n}=z_{n}$ for $1 \leq n \leq N$, and $u_{n}=0$ for $n>N$. In this case also we have

$$
S(Z, u, \rho)=Z \cap[S(Z, 0, \rho)+u] .
$$

For the left side clearly includes the right side, while if $y \in S(Z, u, \rho)$, then for $1 \leq n \leq N$ we have

$$
\sum_{j=n}^{\infty}\left(y_{j}-u_{j}\right)^{2} \leq \sum_{j=1}^{\infty}\left(y_{j}-u_{j}\right)^{2}<\rho^{2} \leq f(n) .
$$

For $n>N$ we have

$$
\sum_{j=n}^{\infty}\left(y_{j}-u_{j}\right)^{2}=\sum_{j=n}^{\infty} y_{j}^{2} \leq f(n),
$$

so that

$$
y \in Z \cap[S(Z, 0, \rho)+u],
$$

and hence

$$
S(Z, u, \rho) \subseteq Z \cap[S(Z, 0, \rho)+u]
$$

for equality. Finally since $z \in S(Z, u \rho)$ by

$$
\|z-u\|=\left(\sum_{j=N+1}^{\infty} z_{j}^{2}\right)^{1 / 2} \leq \sqrt{f(N+1)}<\rho,
$$

we have

$$
z \in S(Z, u, \rho) \subseteq Z \cap[V+u]
$$

so that 


$$
z \in \operatorname{int}(Z \cap[V+u])
$$

$S(Z, u, \rho)$ being open, for Condition i ).

Thus $X$ and $Y$ are special translation spaces, so the result we shall obtain for translation spaces applies to them. For the general translation space $Z$ we define the Borel class $B$ as the $\sigma$-algebra generated by the open subsets of $Z$, given by the relative topology from $R$. For a Borel measure $\phi$ defined over $B$ we note that Condition I of nontriviality and $\mathrm{II}^{\prime}$ of translation-invariance still make perfect sense in this more general context, if $u \in \ell_{2}$ in II' $^{\prime}$ is replaced by $u \in R$. We shall now establish that a locally compact translation space does possess something like a Haar measure, that is a nontrivial, translation-invariant, regular Borel measure. First we need a few more lemmas.

LEMma 8. If $V \subseteq W$ are both open subsets of the translation space $Z$ and if $[W+u] \cap Z$ is open in $Z$ for some $u \in R$, then so also is $[V+u] \cap Z$.

Proof. Since $Z$ is a translation space, it is closed in $R$, so $Z-W$ and $Z-V$ are both closed in $R$ as well as in $Z$. Since open and closed subsets of the topological group $R$ remain such under translation, $B=[(Z-W)+u] \cap Z$ and $C=[(Z-V)+u] \cap Z$ are both closed in $R$, and hence in $Z$. Defining $A=$ $(R-[Z+u]) \cap Z$, we have

$$
A \cup B=Z-([W+u] \cap Z),
$$

known closed in $Z$, so that $\bar{A}-A \subseteq B$ must follow. We obtain $B \subseteq C$ from $V \subseteq W$, and this makes $\bar{A}-A \subseteq C$; thus $Z-([V+u] \cap Z)=A \cup C$ is closed in $Z$, or $[V+u] \cap Z$ is open, as desired.

Let $[B+C]=\{x+y \mid x \in B$ and $y \in C\}$ and $B^{-}=\{x \mid-x \in B\}$ for the following lemma.

Leмma 9. If the translation space $Z$ has compact subsets $B$ and $C$ with $B \cap C=\phi$, then there exists some Z-neighborhood $V$ of zero so that

$$
\left[B+V^{-}\right] \cap\left[C+V^{-}\right]=\phi .
$$

Moreover, both $[V+z] \cap B \neq \phi$ and $[V+z] \cap C \neq \phi$ are not simultaneously possible for any $z \in R$.

Proof. Since $B$ and $C$ are compact subsets of $Z$, they are also such of the topological group $R$. Thus there exists an $R$-neighborhood $\mathbb{W}$ of zero so that 


$$
\left[B+\mathbb{W}^{-}\right] \cap\left[C+\mathbb{W}^{-}\right]=\phi \text {. }
$$

Hence $V=Z \cap W$, so $V^{-} \subseteq \mathbb{W}^{-}$, gives the first result. If $[V+z] \cap B \neq \phi$ and $[V+z] \cap C \neq \phi$, then $z \in\left[B+V^{-}\right] \cap\left[C+V^{-}\right]=\phi$, a contradiction, which gives the last.

Following Halmos [1, p.252], if $B$ and $C$ are subsets of the translation space $Z$, we let $(C: B)$ denote the least cardinal (thus $\aleph_{0}$ or an integer $\geq 0$ ) of sets $P$ of $z \in R$ such that

$$
C \subseteq \underset{z \in P}{\bigcup}[B+z]
$$

Lemma 10. If $C$ is a compact subset of the translation space $Z$ and $V$ is an open Z-subset containing zero, then $(C: V)<+\infty$.

Proof. By Condition i) we have

$$
C \subseteq \bigcup_{u \in R} \text { int }(Z n[V+u])
$$

an open covering of compact $C$. Thus there exists a finite set $A$ of such $u$ with

$$
C \subseteq \underset{u \in A}{\bigcup} \operatorname{int}(Z \cap[V+u]) \subseteq \bigcup_{u \in A}[V+u]
$$

and hence

$$
(C: V) \leq(\operatorname{card} A)<+\infty
$$

This lemma is the only place where Condition i) is used to get our following main result on the existence of a Ilaar measure.

THЕОВЕМ 11. If $Z$ is a locally compact translation space, then there exists a regular Borel measure $\phi$ on $Z$ which is nontrivial (I) and translation-invariant ( II $^{\circ}$ ).

Proof. Since $Z$ is locally compact, it possesses a neighborhood $V_{1}$ of zero such that $\bar{V}_{1}$ is compact, so $0<\left(\bar{V}_{1}: V\right)<+\infty$ for any other $Z$-neighborhood $V$ of zero, by Lemma 10. Also clearly

$$
(C: V) \leq\left(C: \bar{V}_{1}\right)\left(\bar{V}_{1}: V\right) \leq\left(C: V_{1}\right)\left(\bar{V}_{1}: V\right),
$$

so we may define 


$$
\lambda_{v}(C)=\left(\bar{V}_{1}: V\right)^{-1}(C: V)
$$

and have

$$
0 \leq \lambda_{v}(C) \leq\left(C: V_{1}\right)<+\infty
$$

for any compact subset $C$ of $Z$ and any $Z$-neighborhood $V$ of zero. Following Halmos [1, pp. 254-256], we construct a content $\lambda$ from $\lambda_{v}$. Let $\Omega$ be the Cartesian product of the bounded closed intervals $\left[0,\left(C: V_{1}\right)\right]$ over all compact subsets $C$ of $Z ; \Omega$ is compact by Tychonoff's theorem, and each $\lambda_{v} \in \Omega$. Setting

$$
\Lambda(V)=\left\{\lambda_{w} \mid W \subseteq V, W \text { a } Z \text {-neighborhood of zero }\right\},
$$

we see that $\Omega$ contains by compactness some $\lambda \in \cap_{V} \overline{\Lambda(V)}$, the intersection being over all $Z$-neighborhoods $V$ of zero. As in [1], this function $\lambda(C)$ defined over compact $Z$-subsets $C$ is a content; that is, for subsets $B, C$, and $D$ compact we have

$$
0 \leq \lambda(C) \leq \lambda(B)<+\infty
$$

if $C \subseteq B$, and

$$
\lambda(C \cup D) \leq \lambda(C)+\lambda(D)
$$

with equality if $C \cap D=\phi$ by use of Lemma 9. Also $\lambda\left(\bar{V}_{1}\right)=1$ since $\lambda_{v}\left(\bar{V}_{1}\right)=1$ for any $V$. For translation invariance we note that if $[C+z] \subseteq Z$ for a compact $Z$-subset $C$ and $z \in R$, then $[C+z]$ is also compact, since translation by $z$ is a homeomorphism of $R$ onto $R ;([C+z]: V)=(C: V)$, obviously; and thus $\lambda_{v}([C+z])=\lambda_{v}(C)$ for any neighborhood $V$ makes $\lambda([C+z])=\lambda(C)$.

Let $W$ be any subset of $Z$, define the inner content

$$
\lambda_{*}(\mathbb{W})=\sup \lambda(C)
$$

over compact $C \subseteq W$, and for any subset $E$ define

$$
\phi(E)=\inf \lambda_{*}(W)
$$

over open $Z$ subsets $W \supseteq E$. Restricting $\phi$ to $B$, we see that $\phi$ is a regular Borel measure on $Z$; $\phi$ is nontrivial (I) by

$$
\phi(Z) \geq \phi\left(\bar{V}_{1}\right) \geq \lambda\left(\bar{V}_{1}\right)=1 \text { and } \phi\left(V_{1}\right) \leq \lambda\left(\bar{V}_{1}\right)=1,
$$


( see [1, $53 \mathrm{C}$ and E, p. 234]).

It remains only to show that $\phi$ is translation-invariant ( II ${ }^{\prime}$ ). First

$$
\lambda_{*}([W+z])=\lambda_{*}(\mathbb{W})
$$

for $z \in R$ and any $Z$-subset $W$ having $[W+z] \subseteq Z$. For then compact $C \subseteq W$ has $[C+z] \subseteq Z$ and compact, so $\lambda([C+z]) \equiv \lambda(C)$ and thus $\lambda_{*}([W+z]) \geq$ $\lambda_{*}(W)$. The opposite inequality follows symmetrically to give the result, since any compact $C^{\prime} \subseteq[W+z]$ has $C=\left[C^{\prime}-z\right]$ compact with

$$
C \subseteq \mathbb{W} \subseteq Z \text { and } \lambda(C)=\lambda\left(C^{\prime}\right)
$$

Now if $V$ is an open $Z$-subset then $\phi(V)=\lambda_{*}(V)$ since $\lambda_{*}$ is monotone. Thus If $V$ and $[V+u] \cap Z$ are both open in $Z$, and $u \in R$, then $W \subseteq V$ and $[\mathbb{W}+u]=[V+u] \cap Z$, where $\mathbb{W}=[(] V+u] \cap Z)-u]$ so that

$$
\phi([V+u] \cap Z)=\lambda_{*}\left([W+u]=\lambda_{*}(W) \leq \lambda_{*}(V)=\phi(V)\right.
$$

for part b) of Condition II'.

For part a), assume $A \in B, u \in R$, and $A \subseteq V_{0}$, where $V_{0}$ and $\left[V_{0}+u\right]$ are both open $Z$-subsets. Then for any open $Z$-subset $V \supseteq A$, Lemma 8 with $V^{\prime}=V \cap V_{0}$ and $W^{\prime}=V_{0}$ both open makes $\left[V \cap V_{0}+u\right]$ open also, and we note that

$$
[A+u] \subseteq\left[V \cap V_{0}+u\right] \subseteq\left[V_{0}+u\right] \subseteq Z
$$

Hence

$$
\lambda_{*}\left(\left[V \cap V_{0}+u\right]\right)=\lambda_{*}\left(V \cap V_{0}\right)
$$

makes

$$
\begin{aligned}
\phi(A) & =\inf _{\text {open } V \supseteq A} \lambda_{*}(V)=\inf _{\text {open } V \supseteq{ }_{A}} \lambda_{*}\left(V \cap V_{0}\right) \\
& =\inf _{\text {open } V \supseteq A} \lambda_{*}\left(\left[V \cap V_{0}+u\right]\right) \geq \inf _{\text {open } W \supseteq[A+u]} \lambda_{*}(W)=\phi([A+u]) .
\end{aligned}
$$

Symmetrically, $\phi([A+u]) \geq \phi(A)$ gives $\phi([A+u])=\phi(A)$ for our result.

Presumably results similar to Theorem 11 are true for similar subspaces of non-Abelian topological groups. We have considered only the Abelian case for simplicity and because the interesting examples in analysis are Abelian. 
COROLLARY 12. If

$$
\liminf _{n \rightarrow \infty} f(n)=0
$$

then the space $Y$ is locally compact under coincident metric and product topologies, and $Y$ possesses a regular Borel measure nontrivial (I) and translation invariant ( II') under this topology.

Proof. The coincidence of the topologies and local compactness of $Y$ is trivial from $f(n) \downarrow 0$; and Lemma 7 and Theorem 11 give the rest.

6. Another translation space example. In addition to $X$ and $Y$, we want to give another example of a translation space, still with $R=\ell_{2}$. Let

$$
Z_{1}=\left\{x \in \ell_{2} \mid \sum_{n=1}^{\infty} n^{2 r}\left(x_{n}\right)^{2} \leq M\right\}
$$

for some fixed real $r>0$ and $M>0$, so that clearly $Z_{1}$ is actually compact. Such a space would arise by using Fourier analysis on $L_{2}$-function-spaces in which the $r$ th derivative was subjected to a fixed bound in norm. We shall now show that $Z_{1}$ is a translation space, though our proof seems unnecessarily long.

LEMMA 13. If $u \in Z_{1}$ has $u_{n}=0$ for $n>N$ for some finite $N$, and

$$
\rho N^{r} \leq \frac{1}{2}\left\{\sum_{n=1}^{N} n^{2 r}\left(u_{n}\right)^{2}\right\}^{1 / 2}
$$

for some $\rho>0$, then

$$
Z_{1} \cap\left[S\left(Z_{1}, 0, \rho\right)+u\right]=S\left(Z_{1}, u, \rho\right)
$$

open in $Z_{1}$.

Proof. We only need to show that

$$
S\left(Z_{1}, u, \rho\right) \subseteq Z_{1} \cap\left[S\left(Z_{1}, 0, \rho\right)+u\right]
$$

the opposite inclusion being obvious. Consider any $z \in S\left(Z_{1}, u, \rho\right)$; we need only show $(z-u) \in Z_{1}$. Here $\|z-u\|<\rho$, so

$$
\sum_{n=1}^{N} n^{2 r}\left(z_{n}-u_{n}\right)^{2}<N^{2 r} \rho^{2},
$$


and thus from

$$
\rho N^{r} \leq \frac{1}{2}\left\{\sum_{n=1}^{N} n^{2 r}\left(u_{n}\right)^{2}\right\}^{1 / 2}
$$

we obtain, by Minkowski's inequality,

$$
0<\left\{\sum_{n=1}^{N} n^{2 r}\left(u_{n}\right)^{2}\right\}^{1 / 2}-\rho N^{r}<\left\{\sum_{n=1}^{N} n^{2 r}\left(z_{n}\right)^{2}\right\}^{1 / 2} .
$$

Thus $u_{n}=0$ for $n>N$ and $z \in Z_{1}$ yields

$$
\begin{aligned}
\sum_{n=1}^{\infty} n^{2 r}\left(z_{n}-u_{n}\right)^{2}= & \sum_{n=1}^{N} n^{2 r}\left(z_{n}-u_{n}\right)^{2}+\sum_{n=N+1}^{\infty} n^{2 r}\left(z_{n}\right)^{2} \\
& <\rho^{2} N^{2 r}+\sum_{n=1}^{\infty} n^{2 r}\left(z_{n}\right)^{2}-\sum_{n=1}^{N} n^{2 r}\left(z_{n}\right)^{2} \\
& <\rho^{2} N^{2 r}+M-\left(\left\{\sum_{n=1}^{N} n^{2 r}\left(u_{n}\right)^{2}\right\}^{1 / 2}-\rho N^{r}\right)^{2} \\
& =M-\left(\left\{\sum_{n=1}^{N} n^{2 r}\left(u_{n}\right)^{2}\right\}^{1 / 2}-2 \rho N^{r}\right)\left\{\sum_{n=1}^{N} n^{2 r}\left(u_{n}\right)^{2}\right\}^{1 / 2} \leq M .
\end{aligned}
$$

Thus we have shown that

$$
\sum_{n=1}^{\infty} n^{2 r}\left(z_{n}-u_{n}\right)^{2}<M
$$

so $(z-u) \in Z_{1}$ as desired.

THEOREM 14. $Z_{1}$ satisfies Condition $\left.\mathrm{i}\right)$, and hence is a compact translation space possessing a Haar measure in the sense of Theorem 11.

Proof. We merely need to verify Condition i) for $Z_{1}$. Thus given any open $Z_{1}$-subset $V$ containing zero and any $z \in Z_{1}$, we shall find some $u \in Z_{1}$ and $\rho>0$ so that $S\left(Z_{1}, 0, \rho\right) \subseteq V$ and 


$$
z \in Z_{1} \cap\left[S\left(Z_{1}, 0, \rho\right)+u\right]=S\left(Z_{1}, u, \rho\right)
$$

open in $Z_{1}$, which makes $z \in$ int $\left(Z_{1} \cap[V+u]\right)$, as desired. Here we need consider only $z \neq 0$, since $u=0$ makes $0 \in V=$ int $\left(Z_{1} \cap[V+u]\right)$ for the result if $z=0$. Since $z \neq 0$, we may choose $N$ sufficiently large so that

$$
\beta=\left(\sum_{n=1}^{\infty} n^{2 r}\left(z_{n}\right)^{2}\right)^{-1}\left(\sum_{n=N+1}^{\infty} n^{2 r}\left(z_{n}\right)^{2}\right)
$$

has $0 \leq \beta<1 / 5$, and so that

$$
\frac{\sqrt{M}}{2 N^{r}}<\rho_{1}
$$

for some $\rho_{1}$ such that $S\left(Z_{1}, 0, \rho_{1}\right) \subseteq V$. Let

$$
\rho=\frac{1}{2 N^{r}}\left(\sum_{n=1}^{N} N^{2 r}\left(z_{n}\right)^{2}\right)^{1 / 2},
$$

so

$$
\rho \leq \frac{\sqrt{M}}{2 N^{r}}<\rho_{1} \quad \text { and } S\left(Z_{1}, 0, \rho\right) \subseteq V
$$

Define $u \in Z_{1}$ by $u_{n}=z_{n}$ for $1 \leq n \leq N$ and $u_{n}=0$ for $n>N$. By Lemma 13, we have

$$
Z_{1} \cap\left[S\left(Z_{1}, 0, \rho\right)+u\right]=S\left(Z_{1}, u, \rho\right)
$$

open in $Z_{1}$. Finally to complete the proof we have $z \in S\left(Z_{1}, u, \rho\right)$, for

$$
\begin{aligned}
\|z-u\|^{2} & =\sum_{n=N+1}^{\infty}\left(z_{n}\right)^{2} \leq \frac{1}{N^{2 r}}\left(\sum_{n=N+1}^{\infty} n^{2 r}\left(z_{n}\right)^{2}\right) \\
& =\frac{\beta}{N^{2 r}}\left(\sum_{n=1}^{\infty} n^{2 r}\left(z_{n}\right)^{2}\right)<\frac{1}{N^{2 r}}\left(\frac{1-\beta}{4}\right)\left(\sum_{n=1}^{\infty} n^{2 r}\left(z_{n}\right)^{2}\right) \\
& =\frac{1}{\left(2 N^{r}\right)^{2}}\left(\sum_{n=1}^{N} n^{2 r}\left(z_{n}\right)^{2}\right)=\rho^{2},
\end{aligned}
$$


or $\|z-u\|<\rho$, as desired, since $\beta<(1-\beta) / 4$ from $0 \leq \beta<1 / 5$.

7. Summary of results. We have discussed here the translation spaces

$$
X=\left\{x \in \ell_{2}|| x_{n} \mid \leq h(n)\right\}
$$

and

$$
Y=\left\{x \in \ell_{2} \mid \sum_{j=n}^{\infty} x_{j}^{2} \leq f(n)\right\},
$$

and also

$$
Z_{1}=\left\{x \in \ell_{2} \mid \sum_{n=1}^{\infty} n^{2 r}\left(x_{n}\right)^{2} \leq M\right\}
$$

in $\$ 6$, all being subspaces of real separable Hilbert space. For $X$ under the metric topology we have found (Theorem 3 ) that there exists no nontrivial, translation-invariant ( $\mathrm{II}$ or $\mathrm{II}^{\circ}$ ) Borel measure if

$$
\liminf _{n \rightarrow \infty} h(n)>0
$$

under the product topology we have the same conclusion if $h(n)=+\infty$ infinitely often (Theorem 4). If

$$
\sum^{\infty}[h(n)]^{2}<+\infty
$$

which is equivalent to local compactness, then under the metric topology $X$ has a nontrivial, translation-invariant (II) Borel measure which is unique up to constant factors (Theorem 5 ). For $Y$ under the metric topology

$$
\liminf _{n \rightarrow \infty} f(n)=0
$$

or thus $f(n) \downarrow 0$, is equivalent to local compactness, and necessary and sufficient for the existence of a nontrivial, translation-invariant ( $\mathrm{II}^{\prime}$ ) Borel measure (Theorem 3 and Corollary 12). Also we found (Theorem 12) that any locally compact translation space possesses a nontrivial, translation-invariant ( II $^{\prime}$ ) Borel measure; thus so does $Z_{1}$ (Theorem 14).

It is clear from the foregoing results that local compactness is in general 
the crucial condition for the existence of a nontrivial, translation-invariant Borel measure. This is well known for topological groups [ 5, p. 144], and conjectured for spaces with a group germ (a neighborhood of zero in which group addition is always possible). However, it is to be noted that neither $X$ nor $Y$, when locally compact, nor $Z_{1}$ has a group germ. Thus our results seem to be new, and the concept of a translation-space a fruitful one. In fact the idea of a group germ cannot lead to anything here; for it is not difficult to see that any convex metric subspace of $\ell_{2}$, which is locally compact and contains a group germ under $l_{2}$-vector-addition, must be finite dimensional, hence a subspace of $E_{N}$ and thus trivial. In connection with local compactness it should be noted that our results are not complete for $X$; here if $\sum^{\infty}[h(n)]^{2}=+\infty$ the space is not locally compact under the metric topology and presumably no nontrivial, invariant Borel measure exists. We could only show this if

$$
\lim \inf h(n)>0 \text {, }
$$

which assumes more.

The construction of an invariant measure on subspaces of real separable Hilbert space suggests an attempt to carry over vector analysis from $E_{N}$. In particular, in a later paper the author investigates the relationship between $\ell_{2}$-vector-differentiation $[6$, p. 72] and Fourier transforms over $X$. Here $X$ is a modification of Jessen's torus space [3] and can be made into a group, so standard Fourier theory applies [ 7 or 5$]$.

\section{REFERENCES}

1. P. R. Halmos, Measure theory, van Nostrand, New York, 1950.

2. N. A. Kolmogoroff, Grundbegriffe der Wahrscheinlichkeitsrechnung, Ergebnisse der Math., vol. 2, 1933.

3. B. Jessen, The theory of integration in a space of an infinite number of dimensions, Acta Math. 63 (1934), $249-323$.

4. K. Loewner, Goundzüge einer Inhaltslchre in Hilbertschen Raume, Ann. of Math. 40 ( 1939$), 816-833$.

5. A. Weil, L'integration dans les groupes topologiques, Actualites Sc. and Ind., Paris, 1940.

6. E. Hille, Functional analysis and semi-groups, Amer. Math. Soc. Colloquium Publications, New York, 1948.

7. R. Godement \& H. Cartan, Dualité et analyse harmonique, Ann. Sci. Ecole Norm. Sup. 64 (1947), $79-99$.

\section{UNIVERSITY OF WASHINGTON}

SEATTLE 5, WASHINGTON 



\section{PACIFIC JOURNAL OF MATHEMATICS}

\section{EDITORS}

\section{R. M. RoBINSON \\ University of California Berkeley 4, California}

\author{
*R. P. Dilmorth \\ Califomia Institute of Technology \\ Pasadena 4, California
}

E. F. BeckenbaCh, Managing Editor

University of California

Los Angeles 24, California

${ }^{*}$ During the absence of Herbert Busemann in 1952.

\section{ASSOCIATE EDITORS}

$\begin{array}{llll}\text { R. P. DILWORTH } & \text { P. R. HALMOS } & \text { BØRGE JESSEN } & \text { J. J. STOKER } \\ \text { HERBERT FEDERER } & \text { HEINZ HOPF } & \text { PAUL LÉVY } & \text { E. G. STRAUS } \\ \text { MARSHALL HALL } & \text { R. D. JAMES } & \text { GEORGE PÓLYA } & \text { KÖSAKU YOSIDA }\end{array}$

\section{SPONSORS}

UNIVERSITY OF BRITISH COLUMBIA

CALIFORNIA INSTITUTE OF TECHNOLOGY

UNIVERSITY OF CALIFORNIA, BERKELEY

UNIVERSITY OF CALIFORNIA, DAVIS

UNIVERSITY OF CALIFORNIA, LOS ANGELES

UNIVERSITY OF CALIFORNIA, SANTA BARBARA

OREGON STATE COLLEGE

UNIVERSITY OF OREGON

\author{
UNIVERSITY OF SOUTHERN CALIFORNIA \\ STANFORD UNIVERSITY \\ WASHINGTON STATE COLLEGE \\ UNIVERSITY OF WASHINGTON \\ AMERICAN MATHEMATICAL SOCIETY \\ NATIONAL BUREAU OF STANDARDS, \\ INSTITUTE FOR NUMERICAL ANALYSIS
}

Mathematical papers intended for publication in the Pacific Journal of Miathematics should be typewritten (double spaced), and the author should keep a complete copy. Manuscripts may be sent to any of the editors. All other communications to the editors should be addressed to the managing editor, E. F. Beckenbach, at the address given above.

Authors are entitled to receive 100 free reprints of their published papers and may obtain additional copies at cost.

The Pacific Journal of Mathematics is published quarterly, in March, June, September, and December, by the University of California, Berkeley 4, California. The price per volume (4 numbers) is \$8.00; single issues, $\$ 2.50$. Special price to individual faculty members of supporting institutions and to individual members of the American Mathematical Society: $\$ 4.00$ per volume; single issues, $\$ 1.25$.

Subscriptions, orders for back numbers, and changes of address should be sent to the publishers, University of California Press, Berkeley 4, California.

Printed at Ann Arbor, Michigan. Entered as second class matter at the Post Office, Berkeley, California.

\section{UNIVERSITY OF CALIFORNIA PRESS • BERKELEY AND LOS ANGELES}




\section{Pacific Journal of Mathematics}

\section{Vol. 2, No. $4 \quad$ April, 1952}

Shmuel Agmon, On the singularities of Taylor series with reciprocal coefficients .................................... 431

Richard Arens, A generalization of normed rings ............... 455

Iacopo Barsotti, Intersection theory for cycles of an algebraic variety . . . . 473

Leonard M. Blumenthal, Two existence theorems for systems of linear inequalities ..................................... 523

Frank Herbert Brownell, III, Translation invariant measure over separable Hilbert space and other translation spaces................. 531

J. W. S. Cassels, On a paper of Niven and Zuckerman ............... 555

Nelson Dunford, Spectral theory. II. Resolutions of the identity .......... 559

Eugene Lukacs and Otto Szász, On analytic characteristic functions ...... 615

W. A. Mersman, Evaluation of an integral occurring in servomechanism theory.......................................... 627

Lawrence Edward Payne and Alexander Weinstein, Capacity, virtual mass, and generalized symmetrization......................... 633

Choy-Tak Taam, The boundedness of the solutions of a differential equation in the complex domain ...................................... 643 